\title{
Effect of zinc foliar application and mycorrhizal inoculation on morpho-physiological traits and yield parameters of two barley cultivars
}

\author{
Narjes Moshfeghi, ${ }^{1}$ Mostafa Heidari, ${ }^{1}$ Hamid Reza Asghari, ${ }^{1}$ Mehdi Baradaran Firoz Abadi, ${ }^{1}$ \\ Lynette K. Abbott, ${ }^{2}$ Yinglong Chen ${ }^{2}$ \\ ${ }^{1}$ Department of Agronomy and Plant breeding, Faculty of Agriculture, Shahrood University of Technology, \\ Shahrood, Iran; ${ }^{2}$ School of Agriculture and Environment, UWA Institute of Agriculture, The University \\ of Western Australia, Perth WA, Australia
}

\begin{abstract}
Zinc (Zn) plays a vital role in biological systems. Plants require an appropriate balance of this essential micronutrient for growth and optimum yield. This study focused on the effectiveness of foliar application of $\mathrm{Zn}$ combined with inoculation with arbuscular mycorrhizal (AM) fungi on morphological, physiological traits and yield parameters of barley cultivars during the 2015-2016 growing season. In this factorial experiment, different forms of foliar applied $\mathrm{ZnO}$ (nil, nano $\mathrm{Zn}$, ordinary $\mathrm{Zn}$ and nano+ordinary $\mathrm{Zn}$ ) and inoculation with AM fungi (nil, Glomus mosseae and Rhizophagus irregularis) were investigated for two barley cultivars (Yusuf and Julgeh). The two cultivars differed in response to the form of foliar $\mathrm{Zn}$ applied and inoculation with the two commercial inocula of AM fungi. The major responses were significant increases in chlorophyll content $(107 \%)$, soluble sugar $(227 \%)$, grain $\mathrm{Zn}$ concentration $(217 \%)$, carbonic anhydrase activity $(128 \%)$ and grain phytase activity (65\%) for cultivar Julgeh inoculated with G. mosseae when sprayed with nano $\mathrm{ZnO}$ compared with control. Cultivar Julgeh
\end{abstract}

Correspondence: Narjes Moshfeghi, Shahrood University of Technology, Shahrood, Iran.

Tel.: +98.9158841228. E-mail: narjes.moshfeghi@gmail.com

Key words: Arbuscular mycorrhiza; grain $\mathrm{Zn}$; Hordeum vulgare; root characteristics; zinc fertilisation.

Acknowledgements: the Ministry of Science, Research and Technology of Iran provided a short-term research opportunity to NM in support of her study leave at The University of Western Australia.

Contributions: the authors contributed equally.

Conflict of interests: the authors declare no potential conflict of interest.

Received for publication: 3 November 2018.

Revision received: 14 March 2019.

Accepted for publication: 15 March 2019

(C) Copyright N. Moshfeghi et al., 2019

Licensee PAGEPress, Italy

Italian Journal of Agronomy 2019; 14:1354

doi:10.4081/ija.2019.1354

This article is distributed under the terms of the Creative Commons Attribution Noncommercial License (by-nc 4.0) which permits any noncommercial use, distribution, and reproduction in any medium, provided the original author(s) and source are credited. inoculated with $G$. mosseae had physiological traits more likely to enhance productivity and economical yield than did cultivar Yusuf that invested more in root traits and vegetative growth. Consequently, the nano form of $\mathrm{Zn}$ positively increased root and shoot morphological parameters, physiological parameters and grain $\mathrm{Zn}$ concentration, but the ordinary form of $\mathrm{Zn}$ enhanced yields and yield parameters. While foliar $\mathrm{Zn}$ application and inoculation with AM fungi significantly enhanced all measured parameters, the forms of $\mathrm{Zn}$ and inoculation with the two different AM fungi differed in their effectiveness.

\section{Introduction}

Zinc $(\mathrm{Zn})$ is a crucial micronutrient that plays a principal role in growth and development of plants and animals as a co-factor for more than 300 enzymes involved in the metabolism of carbohydrates, lipids, proteins, and nucleic acids (Sadeghzadeh, 2013). It is estimated that construction of about 3000 proteins in the human body is dependent on the presence of $\mathrm{Zn}$ (Andreini et al., 2006). The main reasons for $\mathrm{Zn}$ deficiency in biological systems are the lack of this nutrient in the soil (e.g. in Zn-deficient soil), its limited solubility in soil and low levels in grain (Fileppi et al., 2010). Many physiological perturbations resulting from $\mathrm{Zn}$ deficiency are associated with disruption of enzyme activity, thus Zn-deficiency induced inhibition of photosynthesis is coincident with a decrease in activity of key photosynthetic enzymes (Brown et al., 1993). Carbonic anhydrase, which is present in all photosynthetic tissues and is required for chlorophyll biosynthesis, has a requirement for Zn (Rehman et al., 2012). Therefore, Zn deficiency can reduce rate of photosynthesis due to a sharp decline in carbonic anhydrase activity (Barman et al., 2018). However, Zn efficient crop genotypes with high ability to mobilisation, uptake, utilisation and translocation of $\mathrm{Zn}$ under low $\mathrm{Zn}$ availability (Chaab et al., 2011) have potential to produce more dry matter and grain yield by absorbing more $\mathrm{Zn}$ from $\mathrm{Zn}$-deficient soils (Sundaram and Stalin, 2016). Cultivation of high yielding wheat and barley can lead to a gradual increase in nutrient deficiency, including $\mathrm{Zn}$ deficiency, in plants with a direct negative impact on quality and quantity of crop production (Cakmak, 2009). Hence, there is a need to identify $\mathrm{Zn}$ efficient crop genotypes with high $\mathrm{Zn}$ utilisation efficiency (Singh and Singh, 2011). Interactions between $\mathrm{Zn}$ and other nutrients in soil, especially phosphorus (P), can also increase $\mathrm{Zn}$ deficiency in soil (Imran et al., 2016) and during transmission from root to shoot (Zhang et al., 2016). In a study by Zhang et al. (2012a), P applications greater than $200 \mathrm{~kg} \mathrm{ha}^{-1}$ resulted in significantly increased $\mathrm{P}$ content in different wheat tissues, although the concentration of $\mathrm{Zn}$ in wheat grain was 
reduced. To address the reduction in grain $\mathrm{Zn}$ concentration for high levels of $\mathrm{P}$ application, Zhang et al. (2012a) recommended foliar $\mathrm{Zn}$ application to reduce the $\mathrm{P} / \mathrm{Zn}$ molar ratio in wheat and consequently increase $\mathrm{Zn}$ bioavailability.

Foliar application of $\mathrm{Zn}$ has potential to increase $\mathrm{Zn}$ concentration in wheat grain without soil interactions restricting $\mathrm{Zn}$ availability (Velu et al., 2014; Deshpande et al., 2018) because of its greater efficiency in grain $\mathrm{Zn}$ accumulation in comparison to soil application (Jan et al. 2016). It is a strategy for providing adequate $\mathrm{Zn}$ nutrition in wheat because $\mathrm{Zn}$ is highly mobile in phloem and can be effectively transported from leaves and stems to developing grain (Haslett et al., 2001). Zhang et al. (2012b) showed that grain $\mathrm{Zn}$ concentrations in wheat were increased by $73 \%$ with foliar application of $\mathrm{ZnSO}_{4}$. Similarly, foliar $\mathrm{Zn}$ application was more effective for increasing $\mathrm{Zn}$ concentrations in maize, rice and wheat grain compared to soil application of Zn (Joy et al., 2015). It could also be effective for barley in curbing element imbalance, especially in semi-arid areas where the intensive crop production are annually exposed to high applications of $\mathrm{P}$ fertilisers with potential micronutrient deficiency in grain (Jalali, 2007).

The use of nano fertilisers in foliar applications is an emerging technology with potential benefits compared to soil-applied Zn fertilisers because of more rapid $\mathrm{Zn}$ absorption and reduced $\mathrm{Zn}$ leaching (Morales-Diaz et al., 2017). In a recent study on barley (Janmohammadi et al., 2016) some priorities for increasing crop production using nano-micronutrient fertilisers included their high efficiency, easy application and greater convenience. Nano fertilisers have potential to elevate solubility and mobility of poorly soluble nutrients in soils and increase their bioavailability (Naderi et al., 2013) due to the fact that they can enable nutrients to diffuse more slowly than ordinary fertilisers (Janmohammadi et al., 2016) with increased nutrient use efficiency (Wang et al., 2013).

Arbuscular mycorrhizal (AM) fungi can have a positive influence on plant physiology as well as on root architecture (Dhawal et al., 2016). AM fungi can enhance $\mathrm{P}$ and $\mathrm{Zn}$ absorption by host roots (Thompson et al., 2013) but application of $\mathrm{P}$ fertiliser can reduce $\mathrm{Zn}$ uptake associated with a reduction of mycorrhizal colonisation of roots (Teng et al., 2013). Increasing the availability of $\mathrm{P}$ in soil can negatively affect mycorrhizal colonisation, root $\mathrm{Zn}$ uptake and concentrations of $\mathrm{Zn}$ in wheat tissues (Ova et al., 2015). According to Nouri et al. (2014), the access to Pi is a systemic affinity that depends on the nutritional status of their host plant. AM fungi supply the host with various mineral nutrients in exchange for host assimilates so that the interruption in this symbiosis by high Pi levels, can be attributed as energy saving process for host plant without the loss of assimilates (Smith et al., 2009). Although this symbiotic limitation may occur at the expense of absorption of other elements (Blanke et al., 2005), a meta-analysis of field studies of inoculation of wheat with AM fungi can be an effective agronomic practice (Pellegrino et al., 2015).

Clearly, several factors can combine to influence the $\mathrm{Zn}$ concentration in grain. While there is potential for $\mathrm{Zn}$ and $\mathrm{P}$ fertilisers to interact and reduce availability of $\mathrm{Zn}$ in soil, foliar application of $\mathrm{Zn}$ fertiliser can avoid this problem. Furthermore, the alleviation of potential interactions between $\mathrm{Zn}$ and $\mathrm{P}$ in soil by foliar application of $\mathrm{Zn}$ can be complemented by colonisation of roots by AM fungi to support effective use of P fertiliser. Therefore, the combined effects of foliar application of different forms of $\mathrm{Zn}$ and inoculation with commercial inocula of AM fungi were investigated for two barley cultivars. The hypotheses were: i) that the nano form of $\mathrm{ZnO}$ would be more effective at increasing $\mathrm{Zn}$ concentration in barley grain than the ordinary form of $\mathrm{ZnO}$, due to its smaller size and ease of uptake from foliar application; ii) that barley cultivars would differ in response to both the form of $\mathrm{Zn}$ applied to barley leaves and commercial inocula of AM fungi due to their diverse performance and variation in physiological, phenotypical traits; and iii) that there could be an interaction between the form of foliar Zn application and effect of AM fungal inoculation for the two barley cultivars studied here.

\section{Materials and methods}

\section{Experimental design and planting}

The experiment was a factorial randomised complete design of two barley (Hordeum vulgare L.) cultivars (Yusuf and Julgeh), three AM fungal treatments and four forms of $\mathrm{Zn}$ application; there were three replicates of each treatment. It was conducted in pots placed under field conditions at the Agricultural and Governmental Research Centre of Chenaran, Iran $\left(36^{\circ} 61^{\prime} \mathrm{N}, 59^{\circ} 16^{\prime} \mathrm{E}\right.$, altitude $1221 \mathrm{~m}$ ) during the 2015-2016 cropping season. To make growing conditions much more similar to natural field conditions, the pots were placed in a field plot.

The three AM fungal treatments were: inoculation with commercial inocula of either Glomus mosseae or Rhizophagus irregularis and a control without inoculation. The commercial inocula of AM fungi were obtained from Turan Biotech, Shahrood. The inoculum consisted of a mixture of spores containing soil (with a density of 150 in $100 \mathrm{~g}$ of dry clay). The four foliar $\mathrm{Zn}$ oxide $(\mathrm{ZnO})$ applications were: a control (NO Zn), Nano Zn $\left(2 \mathrm{~g} \mathrm{~L}^{-1}\right)$, Ordinary $\mathrm{Zn}\left(2 \mathrm{~g} \mathrm{~L}^{-1}\right)$ and Nano $\mathrm{Zn}\left(1 \mathrm{~g} \mathrm{~L}^{-1}\right)+$ Ordinary $\left.\mathrm{Zn}\left(1 \mathrm{~g} \mathrm{~L}^{-1}\right)\right]$. Both the ordinary $\mathrm{ZnO}$ particles with a larger diameter (average $200 \mathrm{~nm}$ ) and nano $\mathrm{Zn}$ particles with a smaller diameter (average $20 \mathrm{~nm}$ ) were obtained from Pioneer Nanomaterials Company of Iran.

Plastic pots $(30 \mathrm{~cm}$ top diameter $\times 30 \mathrm{~cm}$ height $)$ were filled with $10 \mathrm{~kg}$ of field soil in a mixture of sand $(3: 1, \mathrm{v}: \mathrm{v})$. Soil was collected from a research farm located at the Agricultural and Governmental Research Centre of Chenaran, Iran. The soil was classified as sandy loam with the following characteristics: $\mathrm{pH} 7.8$, organic matter $1.11 \%$, Zn 0.11 ppm, P 70 ppm, Fe 2.1 ppm, K 550 ppm, $\mathrm{Cu} 0.5 \mathrm{ppm}$. Barley seeds were provided by the Centre of Agriculture and Natural Resources of Mashhad, Iran. Inoculum of AM fungi was applied at the time of sowing. The commercial mycorrhizal inocula was used according to the methods recommended by the company. After creating grooves of approximately $10 \mathrm{~cm}$ in depth within pots, a $2 \mathrm{~cm}$ layer of inoculum $(10 \mathrm{~g})$ was placed in the grooves manually and covered with two centimetres of soil. Six surface sterilised seeds pet pot were placed in the appropriate place on the soil and covered with four centimetres of soil.

Seedlings were thinned to four following emergence. Pots were placed in the field to approximate field growing conditions, with temporarily use of a rain shade in winter to protect against hail damage. All pots were subjected to natural solar radiation and irrigated to $100 \%$ soil water capacity (WC) (Paech and Simonis, 1952) daily by adding the required volume of water after weighing. To calculate $100 \% \mathrm{WC}$, the three pots were filled with soil and water were added till saturation. 6 hours were spent till water had drained by gravity and then weighted. After that soil was dried for two days in compartment dryer at $105^{\circ} \mathrm{C}$ and again weighted. Finally, $100 \% \mathrm{WC}$ is calculated out of the weight of saturation soil minus the dry weight. Zn was applied to barley leaves using a hand sprayer until they were completely covered with the solution. Foliar spraying with the $\mathrm{ZnO}$ treatments was carried out twice; the first spray occurred at tillering and the second spray occurred when 
the grains were at milk stage. Sprays of the different forms of $\mathrm{ZnO}$ were applied at the same concentration of $\mathrm{Zn}$ while for the control (No Zn) treatment, pots were merely sprayed with water.

\section{Physiological and yield measurements}

All plants were harvested at physiological maturity (180 days after planting) and were removed from soil then shoots and roots were separated and dried at $70^{\circ} \mathrm{C}$ for $24 \mathrm{~h}$. Shoot traits [plant height, flag leaf area, leaf area, leaf area:root area ratio, shoot dry weight, and leaf dry weight] and yield components [tiller number, spike number, number of grains plant ${ }^{-1}$, thousand kernel weight, grain yield, harvest index, and straw yield] were recorded. For quantification of physiological parameters and enzyme activities in plants, three fresh leaves of each plant per replicate were collected two weeks after the second Zn application concurrent with physiological maturity at harvesting time. Chlorophyll content was assessed according to Porra et al. (1989), soluble sugar was assessed according to Yang et al. (2001), enzyme carbonic anhydrase was assessed according to the method of Gibson and Leece (1981), phytase activity was assessed according to Barrientos et al. (1994) after recording morphological parameters of harvested leaves. The concentration of $\mathrm{Zn}$ in grain was determined using the method of Chapman and Pratt (1961). To estimate Zn concentration in grain, all samples well washed and properly hulled in order to eliminate possible residential of $\mathrm{Zn}$ in the surface of grains. Grain samples were ground and digested with a boiling acid mixture $\left(\mathrm{HNO}_{3}+\mathrm{HClO}_{4}\right)$ then the concentrations of $\mathrm{Zn}$ in the digest were determined on an ARL 3520 inductively coupled plasma. To measure underground section, roots were thoroughly washed with tap water to remove soil prior to assessing the following root traits: total root length, root area, average root diameter, root volume, root depth, root dry weight, root:shoot dry weight ratio (R:S). Leaf area was measured using a Scanject Delta Scanner. Root parameters were measured using Delta T Scan Software (DELTA SCAN, England) and root images were analysed using WinRHIZO Pro software.

\section{Data analysis}

The experiment was set up in a completely randomised design with factorial arrangement of treatments and three replications. Data were analysed by one-way analysis of variance (ANOVA) using the statistical software SAS (version 9.1). The treatment mean values were compared by least significant difference test at 0.05 level of probability. Data on morphological and physiological traits and yield were subjected to a three-factor ANOVA (factorial $2 \times 3 \times 4)$. Pearson correlation coefficients were calculated for the five physiological traits, along with grain yield and yield parameters were implemented by using SAS software.

\section{Results}

\section{Physiological responses}

Chlorophyll, soluble sugar, carbonic anhydrase and grain phytase activity were all significantly influenced by treatments $(\mathrm{P}<0.01$, Table 1). In both barley cultivars, physiological traits were markedly enhanced following inoculation with AM fungi and foliar Zn spraying (Figures 1 and 2). Physiological traits of cultivar Julgeh inoculated with $G$. mosseae and Yusuf inoculated with $R$. irregularis while both were sprayed with nano $\mathrm{ZnO}$ were significantly affected (Figures 1 and 2). Positive correlations between physiological traits carbonic anhydrase and grain phytase activity and the majority of yield parameters (tiller number, spike number, grain number, thousand kernel weight, harvest index and straw yield) were highly significant $(\mathrm{P}<0.01)$.

\section{$\mathrm{Zn}$ in grain}

$\mathrm{Zn}$ in grain was significantly affected by mycorrhizal inoculation and $\mathrm{Zn}$ spraying $(\mathrm{P}<0.001)$ and was significantly affected by the third order interactions between cultivar $\times$ mycorrhizal inoculation $\times \mathrm{Zn}$ (Table 1). The concentration of $\mathrm{Zn}$ in barley grain was enhanced in both Yusuf and Julgeh following inoculation with AM fungi in combination with foliar $\mathrm{Zn}$ spraying compared with uninoculated plants and lack of $\mathrm{Zn}$ application, but this enhancement was greater in Julgeh than in Yusuf for the same treatments (Figure 2B). All forms of $\mathrm{ZnO}$ increased $\mathrm{Zn}$ concentration in grain with the most marked impact resulting from foliar spraying with nano $\mathrm{ZnO}$ (Figure 2B) compared to the ordinary form of $\mathrm{Zn}$. Increasing the availability of $\mathrm{Zn}$ via foliar application or inoculation with AM fungi enhanced $\mathrm{Zn}$ supply to both barley cultivars.

\section{Shoot and root responses}

Foliar $\mathrm{ZnO}$ treatments and mycorrhizal inoculation significantly affected most shoot and root traits for both barley cultivars $(\mathrm{P}<0.01$, Table 1). Although there were significant second and third order interactions among some traits, they were not signifi-

Table 1. Analysis of variance for root, shoot and physiological traits of two barley cultivars.

\begin{tabular}{|c|c|c|c|c|c|c|c|c|c|c|c|c|c|c|c|c|}
\hline Traits & $\begin{array}{l}\text { TRL } \\
(\mathrm{mm})\end{array}$ & $\begin{array}{c}\mathrm{RA} \\
\left(\mathrm{mm}^{2}\right)\end{array}$ & $\begin{array}{l}\text { ARD } \\
(\mathrm{mm})\end{array}$ & $\begin{array}{c}\mathrm{RV} \\
\left(\mathrm{mm}^{3}\right)\end{array}$ & $\begin{array}{l}\text { RD } \\
(\mathrm{cm})\end{array}$ & $\begin{array}{l}\text { RDW } \\
\text { (g) }\end{array}$ & R/S & $\begin{array}{c}\mathrm{LA} \\
\left(\mathrm{cm}^{2}\right)\end{array}$ & LARA & $\begin{array}{l}\text { SDW } \\
(\mathrm{g})\end{array}$ & $\begin{array}{l}\text { LDW } \\
\text { (g) ( }\end{array}$ & $\begin{array}{c}\text { Chl.T } \\
\left(\mathrm{mg}^{-1} \mathrm{~g} f w\right)\end{array}$ & $\begin{array}{c}\text { SS } \\
\left(\mathrm{mg}^{-1} \mathrm{~g} d w\right)\end{array}$ & $\begin{array}{c}\text { C.A } \\
\text { (units.cm²) }\end{array}$ & $\begin{array}{c}\text { GPH } \\
\left(\mathrm{mg}^{-1} \text { units fw) }\right.\end{array}$ & $\begin{array}{c}\mathrm{G} / \mathrm{n} \\
\left(\mathrm{mg} \mathrm{kg} \mathrm{g}^{-1}\right)\end{array}$ \\
\hline Cultivars (C) & $* *$ & $* *$ & $* *$ & $* *$ & $* *$ & $* *$ & $* *$ & $* *$ & $* *$ & $* *$ & $* *$ & $* *$ & $* *$ & $* *$ & $* *$ & $* *$ \\
\hline Mycorrhiza (M) & $* *$ & $* *$ & $* *$ & $* *$ & $* *$ & $* *$ & $* *$ & $* *$ & $* *$ & $* *$ & $* *$ & $* *$ & $* *$ & $* *$ & $* *$ & $* *$ \\
\hline Zinc (Z) & $* *$ & $* *$ & $* *$ & $* *$ & $* *$ & $* *$ & $* *$ & $* *$ & $* *$ & $* *$ & $* *$ & $* *$ & $* *$ & $* *$ & $* *$ & $* *$ \\
\hline $\mathrm{C} \times \mathrm{M}$ & $* *$ & $* *$ & $* *$ & $* *$ & $* *$ & $* *$ & $* *$ & $* *$ & $* *$ & $* *$ & $* *$ & $* *$ & $* *$ & $*$ & $*$ & $*$ \\
\hline $\mathrm{C} \times \mathrm{Z}$ & $* *$ & ns & ns & $* *$ & ns & ns & ns & $* *$ & $* *$ & $* *$ & $* *$ & $* *$ & $* *$ & $* *$ & $*$ & $* *$ \\
\hline $\mathrm{M} \times \mathrm{Z}$ & $* *$ & $*$ & $*$ & $* *$ & $* *$ & $*$ & $* *$ & $* *$ & $* *$ & $* *$ & $* *$ & $* *$ & $* *$ & ** & ns & $* *$ \\
\hline $\mathrm{C} \times \mathrm{M} \times \mathrm{Z}$ & $* *$ & ns & $* *$ & $* *$ & $* *$ & $*$ & * & $* *$ & $* *$ & $* *$ & $* *$ & $* *$ & $* *$ & ns & ns & $* *$ \\
\hline LSD & 1888 & 4.27 & 0.001 & 0.0006 & 0.19 & 0.002 & 0.001 & 26.88 & 0.0009 & 0.001 & 0.0001 & 0.001 & 0.81 & 1367 & 0.01 & 0.21 \\
\hline
\end{tabular}

TRL, total root length; RA, root area; ARD, average root diameter; RV, root volume; RD, root depth; RDW, root dry weight; R:S, root:shoot ratio; LA, leaf area; LA:RA, ratios of leaf area to root area; SDW, shoot dry weight; LDW, leaf dry weight; Chl. T, chlorophyll total; SS, soluble sugar; CA, carbonic anhydrase; GPH, grain phytase activity; GZn, grain zinc concentration; LSD, least significant difference. *Significant at P<0.05; **significant at $\mathrm{P}<0.01 ;$ ns, not significant. 
cant for cultivar $\times \mathrm{Zn}$ for root area, average root diameter, root depth, root dry weight and root to shoot ratio. Root and shoot morphological traits differed significantly between cultivars (Table 1) and had the lowest values in the absence of application of $\mathrm{ZnO}$ and AM fungi.

Application of nano $\mathrm{Zn}$ frequently showed higher values than other $\mathrm{Zn}$ forms for all root and shoot morphological traits as for the physiological parameters (Table 1). In general, for both barley cultivars, inoculation with AM fungi significantly increased all measured morphological traits of root and shoot (Table 1). Whereas inoculation with $R$. irregularis better illustrated symbiotic responses with cultivar Yusuf, inoculation with $G$. mosseae was more

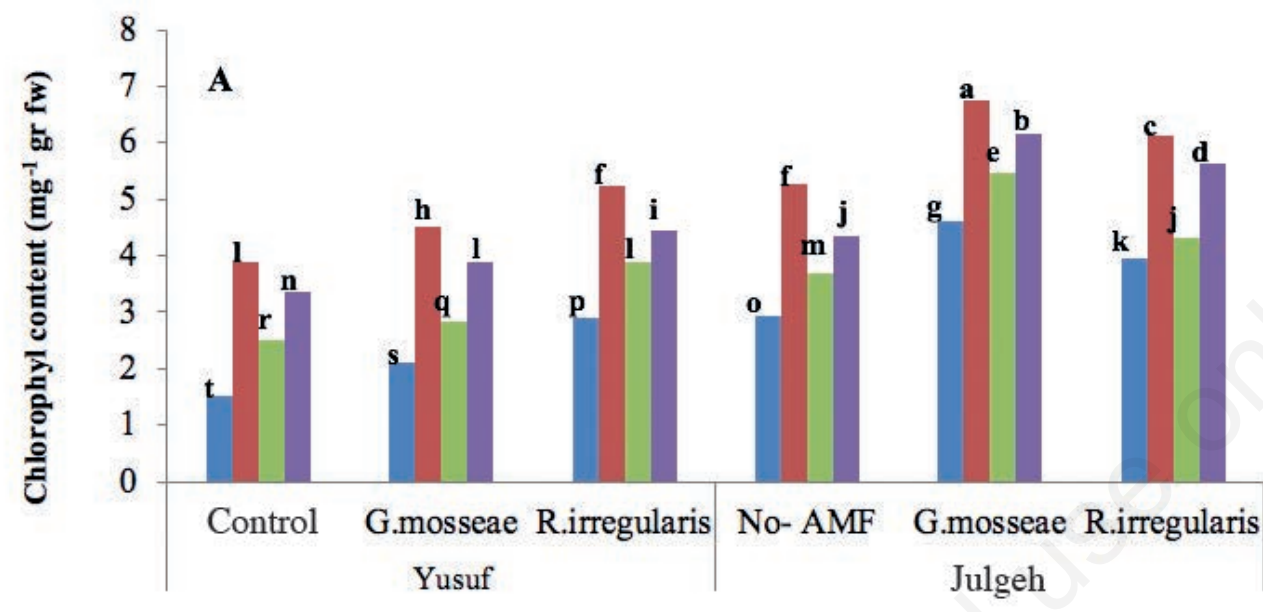

$\sim$ No $\mathrm{Zn}$

- Nano Zn

- Ordinary $\mathrm{Zn}$

- Nano\&Ordinary Zn

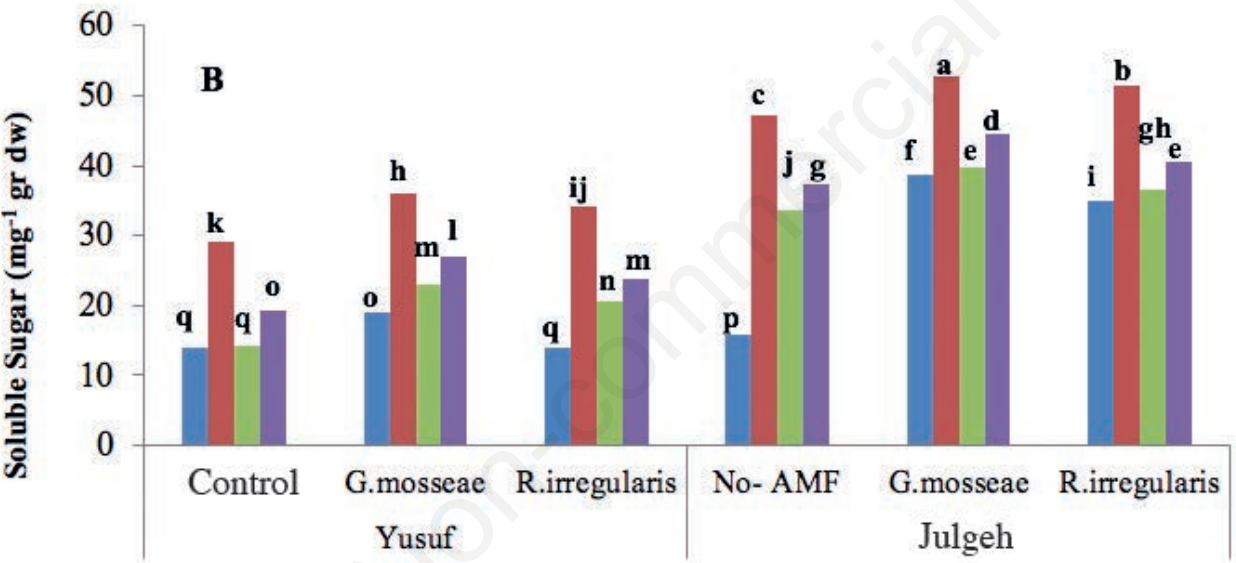

- No $\mathrm{Zn}$

$=$ Nano $\mathrm{Zn}$

= Ordinary $\mathrm{Zn}$

- Nano\&Ordinary Zn

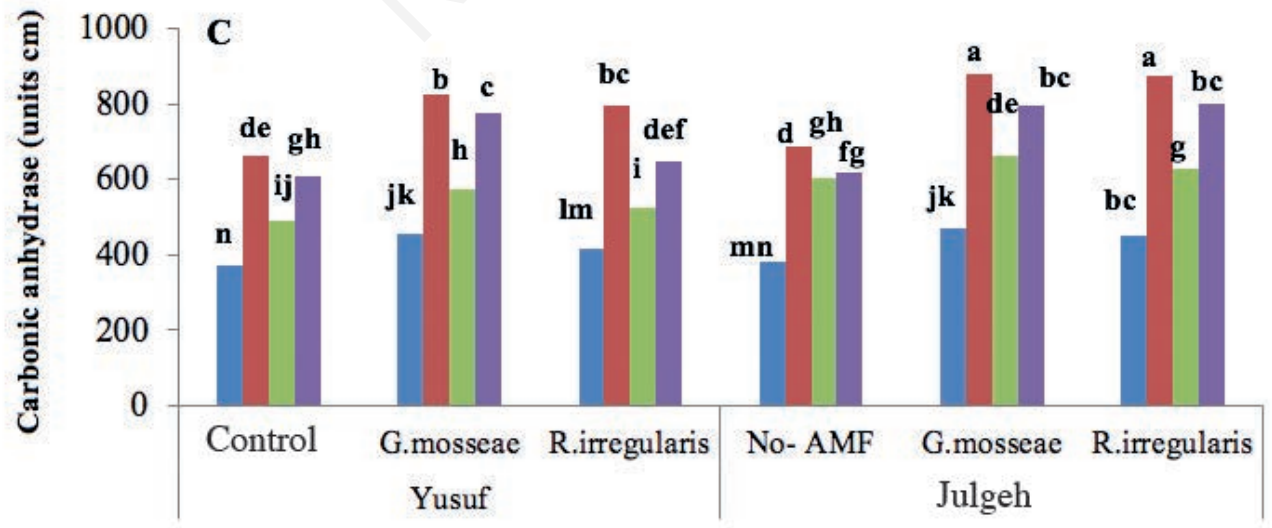

$=$ No $\mathrm{Zn}$

- Nano $\mathrm{Zn}$

$=$ Ordinary $\mathrm{Zn}$

- Nano\&Ordinary Zn

Figure 1. Effects of barley cultivars Yusuf and Julgeh, mycorrhizal inoculation and Zn foliar application on physiological traits chlorophyll total (A), soluble sugar $(B)$ and carbonic anhydrase $(C)$. Different letters represent significant differences $(P<0.05)$. 
effective with the host cultivar Julgeh.

Application of foliar $\mathrm{Zn}$ spray and inoculation with AM fungi significantly increased all yield components in both cultivars. Inoculation with $G$. mosseae significantly increased (63\%) thousand kernel weight, grain yield (128\%) and harvest index (61\%) in cultivar Julgeh compared to non-inoculated Julgeh (Table 2). For cultivar Yusuf, inoculation with $R$. irregularis significantly increased tiller number (104\%), plant height (41\%) and straw yield $(65 \%)$ in comparison with non-inoculated plants (Table 2). However, the effects on traits associated with yield were less consistent, since ordinary $\mathrm{Zn}$ was more effective than nano form to improve yield and yield components.

In addition, correlations between number of spike with grain phytase activity and grain $\mathrm{Zn}$ concentration were significant and positive $\left(r^{2} \geq 0.90\right)$, while grain $\mathrm{Zn}$ concentration was also correlated with tiller number, spike number, grain number, thousand kernel weight, harvest index and straw yield $\left(\mathrm{r}^{2} \geq 0.50\right)$. There were posi- tive and strong correlations between physiological traits and shoot characteristics $\left(\mathrm{r}^{2} \geq 0.50\right)$ (Table 3$)$. Soluble sugar was correlated with harvest index $\left(r^{2}=0.57\right)$ whilst carbonic anhydrase was most strongly correlated with grain phytase activity, grain $\mathrm{Zn}$ and spike number $\left(\mathrm{r}^{2} \geq 0.90\right.$, Table 3$)$. There was also a correlation between grain yield and some yield components (Table 3 ).

Mycorrhizal inoculation resulted in stronger increases in all root morphological traits of both cultivars but these effects differed between cultivars. There were significant increases in root length (95\%), root area $(34 \%)$, root depth $(61 \%)$, root dry weight $(77 \%)$ and shoot dry weight $(190 \%)$ in cultivar Yusuf when compared with the non-inoculated control (Table 4). For cultivar Julgeh, there were fewer significant effects of mycorrhizal inoculation compared with Yusuf, with increases in average root diameter and root volume of $100 \%$ and $211 \%$ respectively. For cultivar Julgeh, inoculation with G. mosseae and spraying with nano $\mathrm{Zn}$ increased leaf area by $18 \%$, leaf area:root area $15 \%$ and leaf dry weight $27 \%$
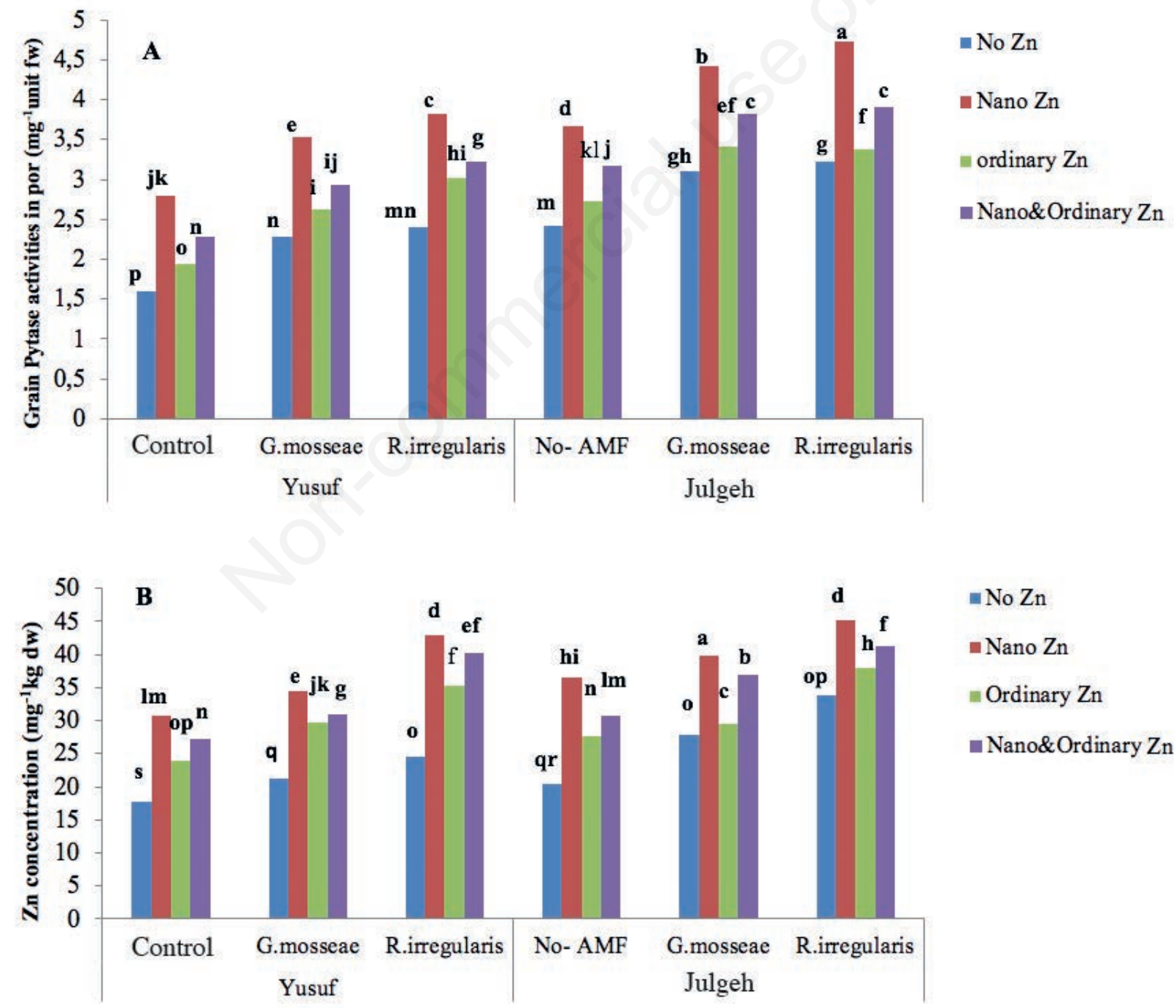

Figure 2. Effects of barley cultivars Yusuf and Julgeh, mycorrhizal inoculation and $\mathrm{Zn}$ foliar application on physiological traits phytase activity (A) and grain zinc concentration (B). Different letters represent significant differences $(P<0.05)$. 
compared with the non-inoculated and non-sprayed control. The highest root:shoot ratios for both cultivars were observed in the controls, which received neither foliar $\mathrm{Zn}$ sprays nor mycorrhizal inoculation. For cultivar Yusuf, there was a positive response in the majority of root morphological traits following inoculation with $R$. irregularis and spraying with nano $\mathrm{Zn}$.

\section{Discussion}

In this study of two barley cultivars, it was shown that: i) application of $\mathrm{Zn}$ whether in the nano or ordinary forms increased the concentration of $\mathrm{Zn}$ in grain, and improved physiological traits and yield parameters; ii) inoculation with AM fungi strongly increased root and shoot morpho-physiological traits, $\mathrm{Zn}$ grain and yield of

Table 2. Effects of foliar $\mathrm{Zn}$ application and inoculation with mycorrhizal fungi on shoot and yield parameters of barley.

\begin{tabular}{|c|c|c|c|c|c|c|c|c|c|c|c|}
\hline Cultivar & Mycorrhiza & $\begin{array}{c}\mathrm{Zn} \\
\text { treatment }\end{array}$ & $\begin{array}{l}\text { TN } \\
\text { (no.) }\end{array}$ & $\begin{array}{c}\text { SN } \\
\text { (no.) }\end{array}$ & GN & $\begin{array}{l}\text { FLA } \\
\left(\mathrm{cm}^{2}\right)\end{array}$ & $\begin{array}{l}\text { PH } \\
(\mathrm{cm})\end{array}$ & $\begin{array}{l}\text { TKW } \\
\text { (g) }\end{array}$ & $\begin{array}{c}\text { GY } \\
\left(\mathrm{g} \cdot \mathrm{pot}^{-1}\right)\end{array}$ & $\begin{array}{l}\text { HI } \\
(\%)\end{array}$ & $\begin{array}{c}\text { SY } \\
\left(\mathrm{g} \mathrm{pot}^{-1}\right)\end{array}$ \\
\hline \multirow[t]{12}{*}{ Yusuf } & \multirow[t]{4}{*}{ Control } & Zn1 & $6.67^{\mathrm{m}}$ & $4.67 \mathrm{p}$ & $153.67^{\mathrm{v}}$ & $9.66^{\mathrm{u}}$ & $87.70^{\mathrm{n}}$ & $27.33^{\mathrm{n}}$ & $5.73^{\mathrm{u}}$ & $23.70^{\mathrm{s}}$ & $18.43^{\mathrm{jk}}$ \\
\hline & & Zn2 & $7.67^{\mathrm{k}}$ & $5.67^{\circ}$ & $168.67^{\mathrm{t}}$ & $11.84^{\mathrm{t}}$ & $91.30^{\mathrm{m}}$ & $33.10^{1}$ & $7.63^{\mathrm{t}}$ & $27.18^{p}$ & $20.43^{\mathrm{i}}$ \\
\hline & & Zn3 & $11.67^{\mathrm{c}}$ & $9.67^{\mathrm{h}}$ & $182.67^{\mathrm{p}}$ & $21.25^{\circ}$ & $105.1^{\mathrm{f}}$ & $38.10^{\mathrm{i}}$ & $10.6^{\mathrm{m}}$ & $34.11^{\mathrm{k}}$ & $20.53^{\mathrm{i}}$ \\
\hline & & Zn4 & $9.67 \mathrm{~g}$ & $7.67^{\mathrm{k}}$ & $174.67^{\mathrm{s}}$ & $17.31 \mathrm{r}$ & $97.30^{\mathrm{j}}$ & $35.70^{\mathrm{j}}$ & $8.63^{r}$ & $29.59^{n}$ & $20.53^{\mathrm{i}}$ \\
\hline & \multirow[t]{4}{*}{ G. mosseae } & Zn1 & $7.67^{\mathrm{k}}$ & $6.67^{\mathrm{m}}$ & $159.67^{\text {u }}$ & $18.24 \mathrm{q}$ & $92.70^{\mathrm{l}}$ & $31.70^{\mathrm{m}}$ & $8.03^{\mathrm{s}}$ & $26.18^{q}$ & $22.63^{\mathrm{h}}$ \\
\hline & & Zn2 & $9.67^{\mathrm{g}}$ & $8.67^{\mathrm{i}}$ & $174.67^{\mathrm{s}}$ & $21.06^{\circ}$ & $98.50^{\mathrm{i}}$ & $37.63^{\mathrm{i}}$ & $9.13^{p}$ & $28.04^{\circ}$ & $23.43^{g}$ \\
\hline & & Zn3 & $12.67^{\mathrm{b}}$ & $11.67^{\mathrm{d}}$ & $188.67^{\mathrm{n}}$ & $32.83^{\mathrm{f}}$ & $116.1^{\mathrm{c}}$ & $42.63^{\mathrm{f}}$ & $12.63^{\mathrm{h}}$ & $33.10^{\mathrm{s}}$ & $25.53^{\mathrm{e}}$ \\
\hline & & Zn4 & $10.67^{\mathrm{e}}$ & $9.67^{\mathrm{h}}$ & $180.67 q$ & $25.90^{\mathrm{l}}$ & $106.9 \mathrm{e}$ & $40.03^{\mathrm{h}}$ & $10.83^{1}$ & $30.80^{\mathrm{m}}$ & $24.33^{\mathrm{f}}$ \\
\hline & \multirow[t]{4}{*}{ R. irregularis } & Zn1 & $8.67^{\mathrm{i}}$ & $7.67^{\mathrm{k}}$ & $176.67^{\mathrm{r}}$ & $27.57^{j}$ & $98.10^{\mathrm{i}}$ & $34.87^{\mathrm{k}}$ & $8.90^{q}$ & $24.60^{r}$ & $27.27^{\mathrm{d}}$ \\
\hline & & Zn2 & $10.67^{\mathrm{e}}$ & $8.67^{\mathrm{i}}$ & $184.67^{\circ}$ & $32.15^{\mathrm{g}}$ & $110.1^{\mathrm{d}}$ & $42.43^{f}$ & $10.70^{\mathrm{m}}$ & $27.31^{p}$ & $28.47^{c}$ \\
\hline & & Zn3 & $13.67^{\mathrm{a}}$ & $11.67^{\mathrm{d}}$ & $202.67^{1}$ & $42.46^{\mathrm{b}}$ & $124.1^{\mathrm{a}}$ & $47.33^{\mathrm{cd}}$ & $13.70^{\mathrm{e}}$ & $31.01^{\mathrm{m}}$ & $30.47^{a}$ \\
\hline & & Zn4 & $11.67^{\mathrm{c}}$ & $10.67^{\mathrm{f}}$ & $195.67^{\mathrm{m}}$ & $36.76^{\mathrm{e}}$ & $117.1^{\mathrm{b}}$ & $44.13^{\mathrm{e}}$ & $12.00^{\mathrm{i}}$ & $29.14^{\mathrm{n}}$ & $29.17^{\mathrm{b}}$ \\
\hline \multirow[t]{13}{*}{ Julgeh } & \multirow[t]{4}{*}{ Control } & Znl & $4.00^{p}$ & $6.00^{\mathrm{n}}$ & $242.67^{\mathrm{k}}$ & $15.14^{\mathrm{s}}$ & $73.43^{\mathrm{s}}$ & $32.83^{\mathrm{l}}$ & $7.70^{\mathrm{t}}$ & $37.75^{j}$ & $12.67^{\circ}$ \\
\hline & & Zn2 & $5.00^{\circ}$ & $8.00^{\mathrm{j}}$ & $268.67^{\mathrm{i}}$ & $19.23^{p}$ & $77.03^{r}$ & $37.37^{\mathrm{i}}$ & $9.30^{\circ}$ & $42.88^{\mathrm{gh}}$ & $12.37^{\circ}$ \\
\hline & & Zn3 & $8.00^{\mathrm{j}}$ & $11.00^{e}$ & $328.67^{\mathrm{k}}$ & $26.86^{\mathrm{k}}$ & $90.83^{\mathrm{m}}$ & $42.37^{\mathrm{f}}$ & $13.30^{\mathrm{f}}$ & $51.19^{b}$ & $12.67^{\circ}$ \\
\hline & & Zn4 & $6.00^{\mathrm{n}}$ & $10.00^{\mathrm{g}}$ & $294.67^{\mathrm{i}}$ & $22.33^{n}$ & $83.03^{p}$ & $39.27^{\mathrm{h}}$ & $11.20^{\mathrm{k}}$ & $46.89^{\mathrm{e}}$ & $12.67^{\circ}$ \\
\hline & \multirow[t]{4}{*}{ G. mosseae } & $\mathrm{Znl}$ & $8.00^{\mathrm{j}}$ & $8.00^{\mathrm{j}}$ & $284.67^{\mathrm{h}}$ & $23.96^{\mathrm{m}}$ & $83.83^{\circ}$ & $40.83^{g}$ & $11.5^{\mathrm{j}}$ & $43.17^{g}$ & $16.30^{\mathrm{m}}$ \\
\hline & & Zn2 & $9.00^{\mathrm{h}}$ & $10.00^{a}$ & $328.67^{\mathrm{e}}$ & $29.71^{\mathrm{i}}$ & $95.83^{\mathrm{k}}$ & $47.63^{c}$ & $13.07^{\mathrm{g}}$ & $47.17^{\mathrm{e}}$ & $17.60^{\mathrm{l}}$ \\
\hline & & Zn3 & $11.00^{\mathrm{d}}$ & $14.00 \mathrm{~g}$ & $384.67^{\mathrm{a}}$ & $47.42^{\mathrm{a}}$ & $109.8^{d}$ & $53.63^{\mathrm{a}}$ & $17.57^{\mathrm{a}}$ & $60.90^{\mathrm{a}}$ & $18.10^{k}$ \\
\hline & & Zn4 & $10.00^{f}$ & $12.00^{c}$ & $346.67^{c}$ & $38.92^{\mathrm{c}}$ & $102.8^{\mathrm{g}}$ & $50.63^{b}$ & $15.07^{\mathrm{c}}$ & $50.65^{c}$ & $18.60^{\mathrm{j}}$ \\
\hline & \multirow[t]{5}{*}{ R. irregularis } & Znl & 5.00 & $7.00^{1}$ & $262.67^{\mathrm{j}}$ & $19.42^{\mathrm{p}}$ & $78.43^{q}$ & $35.33^{\mathrm{k}}$ & $10.27^{\mathrm{n}}$ & $41.47^{\mathrm{i}}$ & $13.50^{\mathrm{n}}$ \\
\hline & & $\mathrm{Zn} 2$ & $7.00^{1}$ & $8.00^{\mathrm{j}}$ & $297.67^{\mathrm{f}}$ & $23.62^{\mathrm{m}}$ & $84.23^{\circ}$ & $42.67^{\mathrm{f}}$ & $12.07^{\mathrm{i}}$ & $42.58^{\mathrm{h}}$ & $13.50^{\mathrm{n}}$ \\
\hline & & Zn3 & $9.00^{\mathrm{h}}$ & $13.00^{\mathrm{b}}$ & $378.67^{\mathrm{b}}$ & $38.44^{\mathrm{d}}$ & $101.8^{\mathrm{h}}$ & $50.67^{\mathrm{b}}$ & $16.37^{\mathrm{b}}$ & $49.23^{\mathrm{d}}$ & $10.50^{\mathrm{p}}$ \\
\hline & & $\mathrm{Zn} 4$ & $7.00^{1}$ & $10.00^{\mathrm{g}}$ & $335.67^{\mathrm{d}}$ & $30.80^{\mathrm{h}}$ & $92.63^{1}$ & $46.67^{\mathrm{d}}$ & $13.87^{\mathrm{d}}$ & $44.73^{f}$ & $13.50^{\mathrm{n}}$ \\
\hline & & LSD $(0.05)$ & 1.3404 & 1.3404 & 5.9946 & 2.5069 & 1.6057 & 1.3623 & 1.2033 & 2.6403 & 0.905 \\
\hline
\end{tabular}

In the same column, values marked with the same letters are similar $(\mathrm{P}<0.05)$, whereas those with different letters are significantly different. $\mathrm{Zn} 1=\mathrm{no}$ spraying $($ control); $\mathrm{Zn} 2=\mathrm{nano} \mathrm{ZnO} ; \mathrm{Zn} 3=$ ordinary $\mathrm{ZnO}$; $\mathrm{Zn} 4=$ nano + ordinary ZnO. TN, tiller number; SN, spike number; GN, number of grains per plant; FLA, flag leaf area; TKW, thousand kernel weight; GY, grain yield; HI, \% harvest index; SY, straw yield; LSD, least significant difference.

Table 3. Correlations coefficients between physiological traits with yield and yield parameters.

\begin{tabular}{|c|c|c|c|c|c|c|c|c|c|c|c|c|c|c|}
\hline & $\begin{array}{c}\text { Chl.T } \\
\left(\mathrm{mg} \mathrm{g}^{-1} \mathrm{fw}\right)\end{array}$ & $\begin{array}{c}\text { SS } \\
\left(\mathrm{mg} \mathrm{g}^{-1} \mathrm{dw}\right)\end{array}$ & $\begin{array}{c}\text { C.A } \\
\text { (units. } \mathrm{cm}^{2} \text { ) }\end{array}$ & $\begin{array}{c}\text { GPH } \\
\text { (Mg/units fw) }\end{array}$ & $\begin{array}{c}\mathrm{G} / \mathrm{n} \\
\left(\mathrm{mg} \mathrm{kg}{ }^{-1}\right)\end{array}$ & $\begin{array}{c}\mathrm{TN} \\
\text { (no.) }\end{array}$ & $\begin{array}{l}\text { SN } \\
\text { (no.) }\end{array}$ & GN & $\begin{array}{l}\text { FLA } \\
\left(\mathrm{cm}^{2}\right)\end{array}$ & $\begin{array}{l}\text { PH } \\
(\mathrm{cm})\end{array}$ & $\begin{array}{l}\text { TKW } \\
(\mathrm{g})\end{array}$ & $\begin{array}{c}\text { GY } \\
\left(\mathrm{g} \mathrm{pot}^{-1}\right)\end{array}$ & $\begin{array}{l}\text { HI } \\
(\%)\end{array}$ & $\begin{array}{c}\text { SY } \\
\left(\mathrm{g} \mathrm{pot}^{-1}\right)\end{array}$ \\
\hline Ch.T & 1.00 & 0.00 & $0.57^{* *}$ & $0.61^{* *}$ & $0.70^{* *}$ & $0.18 \mathrm{~ns}$ & $0.53^{* *}$ & $0.28 * *$ & $-0.52^{* *}$ & $0.24^{*}$ & $0.90^{* *}$ & $0.16 \mathrm{~ns}$ & $0.41^{* *}$ & $0.47^{* *}$ \\
\hline SS & & 1.00 & $0.29 * *$ & $0.28^{* *}$ & $0.13 \mathrm{~ns}$ & $0.45^{* *}$ & $0.17 \mathrm{~ns}$ & $0.35^{* *}$ & $0.45^{* *}$ & $0.56^{* *}$ & $0.24^{*}$ & $0.47^{* *}$ & $0.57^{* *}$ & $0.51^{* *}$ \\
\hline $\mathrm{CA}$ & & & 1.00 & $0.96 * *$ & $0.90^{* *}$ & $0.80^{* *}$ & $0.94 * *$ & $0.83^{* *}$ & $0.01 \mathrm{~ns}$ & $0.48^{* *}$ & $0.66^{* *}$ & $0.47^{* *}$ & $0.70^{* *}$ & $0.58 * *$ \\
\hline $\mathrm{GPH}$ & & & & 1.00 & $0.91^{* *}$ & $0.79^{* *}$ & $0.92 * *$ & $0.78^{* *}$ & $-0.037 \mathrm{~ns}$ & $0.52^{* *}$ & $0.70^{* *}$ & $0.44^{* *}$ & $0.68^{* *}$ & $0.57^{* *}$ \\
\hline GZn & & & & & 1.00 & $0.72 * *$ & $0.89^{* *}$ & $0.72 * *$ & $-0.20 \mathrm{~ns}$ & $0.38 * *$ & $0.71^{* *}$ & $0.27 * *$ & $0.55^{* *}$ & $0.50^{* *}$ \\
\hline NT & & & & & & 1.00 & $0.80^{* *}$ & $0.79^{* *}$ & 0.20 & $0.47^{* *}$ & $0.33^{* *}$ & $0.06^{* *}$ & $0.53^{* *}$ & $0.42 * *$ \\
\hline NS & & & & & & & 1.00 & $0.89 * *$ & $-0.03 \mathrm{~ns}$ & $0.44 * *$ & $0.58 * *$ & $0.42 * *$ & $0.62 * *$ & $0.52 * *$ \\
\hline GN & & & & & & & & 1.00 & $0.18 \mathrm{~ns}$ & $0.50^{* *}$ & $0.40^{* *}$ & $0.49^{* *}$ & $0.63^{* *}$ & $0.51^{* *}$ \\
\hline FLA & & & & & & & & & 1.00 & $0.55^{* *}$ & $-0.18 \mathrm{~ns}$ & $0.64 * *$ & $0.46^{* *}$ & $0.43^{* *}$ \\
\hline $\mathrm{PH}$ & & & & & & & & & & 1.00 & $0.51^{* *}$ & $0.76^{* *}$ & $0.80^{* *}$ & $0.82^{* *}$ \\
\hline TKW & & & & & & & & & & & 1.00 & $0.50^{* *}$ & $0.71^{* *}$ & $0.76^{* *}$ \\
\hline GY & & & & & & & & & & & & 1.00 & $0.90^{* *}$ & $0.88^{* *}$ \\
\hline $\mathrm{HI}$ & & & & & & & & & & & & & 1.00 & $0.93 * *$ \\
\hline SY & & & & & & & & & & & & & & 1.00 \\
\hline
\end{tabular}

Chl.T, chlorophyll total; SS, soluble sugar; CA, carbonic anhydrase; GPH, grain phytase activity; GZn, grain zinc concentration; TN, tiller number; SN, spike number; GN, number of grains per plant; FLA, flag leaf area; PH plant height; TKW, thousand kernel weight; GY, grain yield; HI, \% harvest index; SY, straw yield. *Significant at $\mathrm{P}<0.05$; **significant at $\mathrm{P}<0.01$; ns, not significant. 
barley cultivars; and iii) both barley cultivars responded distinctly to inoculation with AM fungi. However, the commercial inoculum of $G$. mosseae had a better response with cultivar Julgeh and the commercial inoculum $R$. irregularis had a better response with cultivar Yusuf.

\section{The main differences between two barley cultivars}

Our findings illustrated a significantly higher plant yield and yield components following application of $\mathrm{Zn}$ in both investigated barley cultivars, but the increases were greater for cultivar Julgeh. This enhancement in Julgeh yield was expected with based on the responses in physiological and biochemical parameters including the increment in chlorophyll content and carbonic anhydrase activity in comparison with those of Yusuf under the same treatment. Indeed, cultivar Yusuf, displayed a higher potential and invested more in the development of root traits. Zn-deficient barley varieties have previously been shown to have greater root mass compared to shoot mass (Tiong et al., 2015). As in our experiment, this has also been observed for wheat (Rengel and Romheld, 2000; Kumar, 2001). Our findings corresponded with these observations for Yusuf but to a lesser extent for Julgeh, which was the more efficient cultivar, based on higher shoot and yield parameters. Julgeh was more efficient than Yusuf based on the definition of Singh et al. (2005) because of its higher capacity to absorb $\mathrm{Zn}$. We assume that the advantage in increasing the $\mathrm{Zn}$ concentration of Julgeh grain compared to that of Yusuf can also be traced to genetic variation between cultivars, in parallel with observations for wheat cultivars (Rengel and Romheld, 2000). Our findings for barley cul- tivars coincide with those of biochemical traits for wheat genotype with higher $\mathrm{Zn}$ efficiency, where the activity of carbonic anhydrase was two-fold greater than in $\mathrm{Zn}$-inefficient genotypes (Rengel, 1995). Therefore, we hypothesize that the genetic dominance of Julgeh whether in its biochemical traits including the greatest chlorophyll content, carbonic anhydrase and soluble sugar or morphological parameters as shoot development may have led to the enhancement of its yield parameters.

\section{The influence of nano and ordinary form of $\mathrm{Zn}$ foliar application on barley}

Foliar application of $\mathrm{Zn}$ in crop production has been recommended as an effective way to compensate cereal yield and improve nutrient deficiency of this vital element, especially in severely deficient soils (Cakmak, 2009). As previously reported (Khan et al., 2014), interactions between $\mathrm{P}$ and $\mathrm{Zn}$ in the rhizosphere, especially due to the excessive use of $\mathrm{P}$ fertiliser, can result in an imbalance between the amounts of these essential elements in plant tissue, leading to $\mathrm{Zn}$ deficiency in shoots and grain. Therefore, it can be hypothesised that the reduction in crop productivity common in rain-fed and low fertility soils could be addressed with foliar application of nutrients, especially micronutrients, as suggested by Prasad et al. (2012). This was previously supported in a pot experiment (Genc et al., 2004) for two barley genotypes differing in $\mathrm{Zn}$ efficiency growing in deficient sandy soil. With application of different levels of $\mathrm{Zn}$ fertiliser rating from 0-12.8 (mg-1 $\mathrm{kg}$ dry soil), the concentration of $\mathrm{Zn}$ in grain

Table 4. Effects of $\mathrm{Zn}$ foliar application and inoculation with arbuscular mycorrhizal fungi on root and shoot parameters of two barley cultivars.

\begin{tabular}{|c|c|c|c|c|c|c|c|c|c|c|c|c|c|}
\hline Cultivar & Mycorrhiza & $\begin{array}{l}\text { a } \mathrm{Zn} \\
\text { treatment }\end{array}$ & $\begin{array}{l}\text { TRL } \\
(\mathrm{mm})\end{array}$ & $\begin{array}{c}\mathrm{RA} \\
\left(\mathrm{mm}^{2}\right)\end{array}$ & $\begin{array}{l}\text { ARD } \\
(\mathrm{mm})\end{array}$ & $\begin{array}{c}\mathrm{RV} \\
\left(\mathrm{mm}^{3}\right)\end{array}$ & $\begin{array}{c}\mathrm{RD} \\
(\mathrm{cm})\end{array}$ & $\begin{array}{l}\text { RDW } \\
\text { (g) }\end{array}$ & $\mathrm{R} / \mathrm{S}$ & $\begin{array}{c}\mathrm{LA} \\
\left(\mathrm{cm}^{2}\right)\end{array}$ & LA/RA & $\begin{array}{l}\text { SDW } \\
\text { (g) }\end{array}$ & $\begin{array}{l}\text { LDW } \\
\text { (g) }\end{array}$ \\
\hline \multirow[t]{12}{*}{ Yusuf } & \multirow[t]{4}{*}{ Control } & Zn1 & $2593^{1}$ & $215.3^{j \mathrm{k}}$ & $0.61^{\mathrm{k}}$ & $7.2^{q}$ & $57.24^{\circ}$ & $0.74^{1 \mathrm{mn}}$ & $0.74^{\mathrm{a}}$ & $258^{n}$ & $1.20^{\mathrm{kl}}$ & $1.00^{q}$ & $0.47^{\mathrm{m}}$ \\
\hline & & Zn2 & $2946^{\mathrm{i}}$ & $231.3^{\mathrm{h}}$ & $0.92^{\mathrm{hi}}$ & $9.8^{\mathrm{k}}$ & $62.66^{\mathrm{j}}$ & $0.86^{\mathrm{jk}}$ & $0.57^{\mathrm{de}}$ & $295^{1}$ & $1.28^{\mathrm{gh}}$ & $1.51^{\mathrm{m}}$ & $0.76^{\mathrm{g}}$ \\
\hline & & Zn3 & $2673^{\mathrm{k}}$ & $224.0^{\mathrm{i}}$ & $0.64^{\mathrm{k}}$ & $8.5^{\mathrm{n}}$ & $58.81^{\mathrm{n}}$ & $0.78^{\mathrm{klm}}$ & $0.64^{\mathrm{bc}}$ & $260^{\mathrm{n}}$ & $1.16^{l}$ & $1.22^{\circ}$ & $0.53^{\mathrm{k}}$ \\
\hline & & Zn4 & $2806^{j}$ & $228.0^{\mathrm{h}}$ & $0.77^{\mathrm{j}}$ & $9.2^{\mathrm{mn}}$ & $60.59^{1}$ & $0.83^{\mathrm{jkl}}$ & $0.59^{\text {cd }}$ & $282^{m}$ & $1.24^{\mathrm{ij}}$ & $1.40^{\mathrm{n}}$ & $0.63^{\mathrm{i}}$ \\
\hline & \multirow[t]{4}{*}{ G. mosseae } & $\mathrm{Zn} 1$ & $3133^{\mathrm{h}}$ & $238.6^{\mathrm{g}}$ & $0.66^{\mathrm{k}}$ & $7.7^{p}$ & $71.96^{\mathrm{h}}$ & $1.00^{\mathrm{gh}}$ & $0.66^{\mathrm{b}}$ & $295^{1}$ & $1.24^{\mathrm{ij}}$ & $1.76^{\mathrm{j}}$ & $0.63^{\mathrm{i}}$ \\
\hline & & Zn2 & $3740^{\mathrm{e}}$ & $259.3^{\mathrm{d}}$ & $1.01^{\mathrm{f}}$ & $8.7^{\mathrm{m}}$ & $80.58 \mathrm{e}$ & $1.14^{\text {cde }}$ & $0.46^{\mathrm{jk}}$ & $338^{\mathrm{hi}}$ & $1.31^{\mathrm{g}}$ & $2.48^{c}$ & $0.89^{\mathrm{e}}$ \\
\hline & & Zn3 & $3306^{9}$ & $246.0^{\mathrm{f}}$ & $0.80^{\mathrm{j}}$ & $8.2^{\circ}$ & $74.67^{\mathrm{g}}$ & $1.01^{\mathrm{gh}}$ & $0.50^{\mathrm{hi}}$ & $305^{\mathrm{k}}$ & $1.24^{\mathrm{ij}}$ & $2.02^{\mathrm{fg}}$ & $0.77^{\mathrm{g}}$ \\
\hline & & Zn4 & $3526^{\mathrm{f}}$ & $252.0^{\mathrm{e}}$ & $0.91^{\mathrm{hi}}$ & $9.0^{\mathrm{mn}}$ & $78.59^{f}$ & $1.02^{\mathrm{fgh}}$ & $0.45^{\mathrm{jk}}$ & $316^{\mathrm{j}}$ & $1.25^{\mathrm{hi}}$ & $2.25^{\mathrm{d}}$ & $0.82^{f}$ \\
\hline & \multirow[t]{4}{*}{ R. irregularis } & Zn1 & $3906^{\mathrm{d}}$ & $266.6^{c}$ & $0.81^{j}$ & $8.3^{\circ}$ & $84.17^{\mathrm{d}}$ & $1.11^{\text {def }}$ & $0.56^{\mathrm{ef}}$ & $314 j$ & $1.18^{\mathrm{kl}}$ & $1.98^{\mathrm{gh}}$ & $0.43^{n}$ \\
\hline & & $\mathrm{Zn2}$ & $5060^{\mathrm{a}}$ & $288.0^{\mathrm{a}}$ & $1.02^{f}$ & $9.7^{\mathrm{k}}$ & $92.46^{\mathrm{a}}$ & $1.31^{\mathrm{a}}$ & $0.45^{\mathrm{jk}}$ & $368^{\mathrm{ab}}$ & $1.28^{\mathrm{gh}}$ & $2.90^{\mathrm{a}}$ & $0.89^{\mathrm{e}}$ \\
\hline & & Zn3 & $4160^{c}$ & $276.0^{\mathrm{b}}$ & $0.89^{\mathrm{i}}$ & $\begin{array}{l}0.1 \\
8.9^{\mathrm{mn}}\end{array}$ & $85.48^{\mathrm{c}}$ & $1.19^{\mathrm{bcd}}$ & $0.52^{\text {gh }}$ & $336^{\mathrm{hi}}$ & $1.22^{\mathrm{jk}}$ & $2.28^{\mathrm{d}}$ & $0.83^{\mathrm{f}}$ \\
\hline & & Zn4 & $4453^{b}$ & $279.3^{b}$ & $0.95^{\text {gh }}$ & $9.1^{\ln }$ & $88.05^{\mathrm{b}}$ & $1.26^{\mathrm{ab}}$ & $0.47 \mathrm{jk}$ & $347^{\text {ef }}$ & $1.24^{\mathrm{ij}}$ & $2.69^{b}$ & $0.87^{\mathrm{d}}$ \\
\hline \multirow[t]{13}{*}{ Julgeh } & \multirow[t]{4}{*}{ Control } & Zn1 & $1793^{u}$ & $166.6^{q}$ & $0.76^{j}$ & $9.5^{\mathrm{m}}$ & $48.14^{t}$ & $0.63^{\circ}$ & $0.57^{\mathrm{de}}$ & $319^{j}$ & $1.71^{\mathrm{de}}$ & $0.95^{\mathrm{q}}$ & $0.31^{\circ}$ \\
\hline & & Zn2 & $2026^{\mathrm{s}}$ & $182.6^{0}$ & $1.07^{\mathrm{e}}$ & $13.1^{\mathrm{i}}$ & $53.77^{9}$ & $0.69^{\text {no }}$ & $0.44^{\mathrm{kl}}$ & $347^{\mathrm{de}}$ & $1.62^{\mathrm{f}}$ & $1.55^{\mathrm{lm}}$ & $0.62^{\mathrm{ij}}$ \\
\hline & & Zn3 & $1886^{\mathrm{t}}$ & $174.0^{\mathrm{p}}$ & $0.87^{\mathrm{i}}$ & $12.0^{\mathrm{kl}}$ & $49.51^{\mathrm{s}}$ & $0.64^{\circ}$ & $0.56^{\mathrm{de}}$ & $331^{\mathrm{i}}$ & $1.75^{\mathrm{d}}$ & $1.14 \mathrm{p}$ & $0.42^{\mathrm{n}}$ \\
\hline & & Zn4 & $1953^{\mathrm{t}}$ & $180.6^{\circ}$ & $0.99^{f g}$ & $12.2^{\mathrm{jk}}$ & $51.54^{r}$ & $0.65^{\circ}$ & $0.48^{\mathrm{ij}}$ & $345^{\text {fg }}$ & $1.69 \mathrm{e}$ & $1.34^{\mathrm{n}}$ & $0.51^{1}$ \\
\hline & \multirow{4}{*}{ G. mosseae } & $\mathrm{Znl}$ & $2320^{\circ p}$ & $202.0^{1}$ & $1.21^{\mathrm{d}}$ & $27.3^{c}$ & $56.89^{\circ}$ & $0.89^{i j}$ & $0.50^{\mathrm{hi}}$ & $355^{\mathrm{de}}$ & $1.92^{b}$ & $1.76^{\mathrm{j}}$ & $0.74^{\mathrm{h}}$ \\
\hline & & Zn2 & $2493^{\mathrm{m}}$ & $223.3^{\mathrm{i}}$ & $1.53^{\mathrm{a}}$ & $29.6^{\mathrm{a}}$ & $66.00^{\mathrm{i}}$ & $1.23^{\mathrm{abc}}$ & $0.54^{\mathrm{fg}}$ & $377^{\mathrm{a}}$ & $1.98^{\mathrm{a}}$ & $2.27^{\mathrm{d}}$ & $1.15^{\mathrm{a}}$ \\
\hline & & Zn3 & $2380^{\text {no }}$ & $212.6^{\mathrm{k}}$ & $1.32^{c}$ & $26.8^{d}$ & $59.7 \mathrm{~m}^{\mathrm{m}}$ & $0.95^{\mathrm{hi}}$ & $0.49^{\text {ig }}$ & $372^{\mathrm{ab}}$ & $1.90^{\mathrm{b}}$ & $1.94^{\mathrm{h}}$ & $0.90^{\mathrm{d}}$ \\
\hline & & Zn4 & $2440^{\mathrm{nm}}$ & $217.3^{\mathrm{j}}$ & $1.44^{\mathrm{b}}$ & $28.6^{\mathrm{b}}$ & $63.32^{j}$ & $1.08^{\mathrm{efg}}$ & $0.50^{\mathrm{hi}}$ & $366^{\mathrm{bc}}$ & $1.91^{\mathrm{b}}$ & $2.16^{\mathrm{e}}$ & $0.94^{b}$ \\
\hline & \multirow[t]{5}{*}{ R. irregularis } & Znl & $2080^{r s}$ & $183.3^{\circ}$ & $0.89^{\mathrm{i}}$ & $21.4^{\mathrm{h}}$ & $55.41^{\mathrm{p}}$ & $0.72^{\mathrm{mno}}$ & $0.45^{\mathrm{jk}}$ & $336^{\text {hi }}$ & $1.83^{\mathrm{c}}$ & $1.59^{1}$ & $0.83^{f}$ \\
\hline & & Zn2 & $2253^{p q}$ & $195.3^{\mathrm{m}}$ & $1.23^{\mathrm{d}}$ & $24.3^{\mathrm{e}}$ & $61.88^{\mathrm{k}}$ & $0.81^{\mathrm{jkl}}$ & $0.39^{1}$ & $362^{d}$ & $1.93^{\mathrm{b}}$ & $2.06^{\mathrm{f}}$ & $0.73^{\mathrm{h}}$ \\
\hline & & Zn3 & $2126^{r}$ & $188.0^{\mathrm{n}}$ & $0.92^{\mathrm{hi}}$ & $22.5^{\mathrm{g}}$ & $57.28^{\circ}$ & $0.75^{1 \mathrm{mn}}$ & $0.45^{\mathrm{jk}}$ & $343^{\mathrm{gh}}$ & $1.82^{\mathrm{c}}$ & $1.66^{\mathrm{k}}$ & $0.52^{\mathrm{kl}}$ \\
\hline & & Zn4 & $2200^{q}$ & $192.0^{\mathrm{m}}$ & $1.03^{\mathrm{ef}}$ & $23.7^{\mathrm{f}}$ & $59.5 \mathrm{~g}^{\mathrm{m}}$ & $0.77^{1 \mathrm{mn}}$ & $0.42^{\mathrm{kl}}$ & $355^{\mathrm{de}}$ & $1.85^{\mathrm{c}}$ & $1.84^{\mathrm{i}}$ & $0.61^{\mathrm{j}}$ \\
\hline & & LSD $(0.05)$ & 71.349 & 3.3954 & 0.054 & 0.0404 & 0.7302 & 0.0885 & 0.0544 & 8.5128 & 0.0501 & 0.0584 & 0.019 \\
\hline
\end{tabular}

In the same column, values marked with the same letters are similar $(\mathrm{P}<0.05)$, whereas those with different letters are significantly different. $\mathrm{Zn} 1=\mathrm{no}$ spraying; $\mathrm{Zn} 2=$ nano $\mathrm{ZnO} ; \mathrm{Zn} 3=$ ordinary $\mathrm{ZnO}$; $\mathrm{Zn} 4=$ nano + ordinary ZnO. TRL, total root length; RA, root area; ARD, average root diameter; RV, root volume; RD, root depth; RDW, root dry weight; R:S, root:shoot ratio; LA, leaf area; LA:RA, ratios of leaf area to root area; SDW, shoot dry weight; LDW, leaf dry weight; LSD, least significant difference. 
and the grain yield in the more efficient genotype were significantly increased compared to those of the $\mathrm{Zn}$-inefficient genotype. They stated that the improvement in the $\mathrm{Zn}$ efficient cultivar, which is occurred in all level of $\mathrm{Zn}$ fertilisers even under $\mathrm{Zn}$ deficiency, was due to high translocation of $\mathrm{Zn}$ from vegetative plant parts to grain. Similarly, our observation that foliar application of $\mathrm{Zn}$ to barley leaves improved grain $\mathrm{Zn}$ concentration, grain yield, thousand kernel weight and harvest index parameters for Julgeh more than for Yusuf. These differences may be due to genotypic variation between cultivars in their $\mathrm{Zn}$ absorption capability due to differences in leaf structure in favour of Julgeh. These findings were in accordance with a study by Painkra et al. (2015) where the concentration of $\mathrm{Zn}$ in rice grain was significantly influenced by genotypes and Zn. Eleiwa et al. (2013) also reported that foliar application of $\mathrm{Zn}$ influenced barley vegetative growth including plant height, spike length, tillering, leaf number and area, as well as photosynthesis pigments (chlorophyll a, b and carotenoids). Thus, an appropriate concentration of $\mathrm{ZnO}$ has potential to improve growth of barley by promoting physiological parameters as chlorophyll, photosynthesis and essential growth enzymes. Foliar application of $\mathrm{Zn}$ would therefore be a plausible strategy to overcome the lack of $\mathrm{Zn}$ in plants as recently reported by Yagmur et al. (2017) where there was remobilization and loading of $\mathrm{Zn}$ from leaves to grain of barley cultivars following spraying with $\mathrm{Zn}$ under field conditions. We demonstrated that among the forms of $\mathrm{Zn}$ applied as a foliar spray to the two barley cultivars in our experiment, the nano form of $\mathrm{Zn}$ lead to the highest concentrations of chlorophyll and soluble sugar in leaves. This is in line with a previous exploratory study reporting that nanoparticles below100 nm possess high potential to penetrate plant cells through either stomata or the vascular system (Eichert et al., 2008) due to their smaller particle size, in both diameter and weight (Fedorenko et al., 2015), greater solubility in water and more efficiency compared to ordinary forms of $\mathrm{Zn}$ (Joseph and Morrison, 2006). Previous studies also showed that foliar application of $\mathrm{ZnO}$ nanoparticles increased $\mathrm{Zn}$ concentration in grain of maize (Subbaiah et al., 2016) and durum wheat (Deshpande et al., 2018). In our study, the nano form of $\mathrm{Zn}$ also lead to a higher concentration of $\mathrm{Zn}$ in grain and to greater carbonic anhydrase activity in leaves and grain phytase activity for cultivar Julgeh. A significant increase in the root and shoot morphological traits of both barley cultivars with application of the nano compared to ordinary form of $\mathrm{Zn}$ is another characteristic of this novel $\mathrm{Zn}$ fertiliser. Application of $\mathrm{ZnO}$ in the form of nanoparticles $(20 \mathrm{ppm})$ to Cicer ariatium (chickpea) seedlings enhanced root and shoot biomass by about $42 \%$ and $98 \%$ respectively (Mahajan et al., 2011). For Pearl Millet, root area increased with foliar application of nano $\mathrm{Zn}$ fertiliser (Tarafdar et al., 2014). But contradictory results were associated with the yield and yield related parameters of both cultivars indicated that the foliar spraying of $\mathrm{Zn}$ with significant increase in yield and yield component occurred with the advantage of ordinary form compared to the nano. Somewhat different results were obtained by Janmohammadi et al. (2016) for barley in which they found that the foliar application of nano fertiliser in a combination of $\mathrm{Zn}$ nano-chelate with 2000 ppm nano- $\mathrm{TiO}_{2}$ significantly influenced barley traits and yield components compared with the ordinary form when grown in the field. Tarafdar et al. (2014) also reported significant improvement in grain yield and $\mathrm{Zn}$ concentration for Pearl Millet following foliar application of nano $\mathrm{Zn}$. While foliar application of the nano form of $\mathrm{Zn}$ influenced root and shoot growth, and all biochemical traits of barley in our study, it is difficult to understand the basis of a preferential response of yield improvement compared with the ordinary form of $\mathrm{Zn}$ foliar appli- cation. One possibility is that since for improvement of yield and yield components in crop there is a need for use of optimum concentration of nano fertilisers Singh et al. (2017), the application of nano $\mathrm{Zn}$ less than optimum may enable to achieve priority to increase traits related to yield in comparison to ordinary form. Therefore, we hypothesized that optimum applied dose and concentration of $\mathrm{ZnO}$ more than applied doses in this study could enhance the yield parameters as well as morpho-physiological traits. Further research to determine the appropriate dose of foliar application of nano $\mathrm{Zn}$ on the barley plant is required. Nevertheless, it reflects a high potential for optimum doses of $\mathrm{Zn}$ nano fertiliser, which is likely to be environmentally friendly and affordable (Tarafdar et al., 2014) with potential to improve crop production and increase essential micronutrients in grain.

\section{The influence of mycorrhizal inoculation on barley}

In our study, inoculation with commercially available AM fungi, especially $G$. mosseae with Julgeh and $R$. irregularis with Yusuf, positively enhanced the $\mathrm{Zn}$ concentration in barley grain by $192 \%$ and $159 \%$ respectively in plants grown in pots under field conditions. Improvements in grain quality with strategies to enhance the concentration of $\mathrm{Zn}$ in grain lead to better root and shoot traits compared to those arising from low $\mathrm{Zn}$ content in seeds (Cakmak, 2008). This will ensure improvement in seed vigour and seed germination in the next generation (Kinaci and Kinaci, 2005). Furthermore, seedlings that emerge from seeds with a high level of $\mathrm{Zn}$ can reinforce their tolerance and resistance in adverse environmental circumstances (Mousavi, 2011). According to the findings described by Kothari et al. (1991), maize plants inoculated with an AM fungus increased $\mathrm{Zn}$ concentration in shoots by $164 \%$. Similarly, for wheat, mycorrhizal colonization increased with increases in root, shoot and grain $\mathrm{Zn}$ (Zhang et al., 2016). The enhancement in the concentration of $\mathrm{Zn}$ and $\mathrm{P}$ in wheat grain, grain yield and the strong positive correlation between inoculation with AM fungi and the aforementioned traits was revealed from the meta-analysis conducted by Pellegrino et al. (2015).

Improvement in grain quality following inoculation with AM fungi could be due to enhanced nutrient availability or to enhancement of the microbial population in the rhizosphere (Barea et al., 2005). In our study, Yusuf inoculated with $R$. irregularis when it was sprayed with nano $\mathrm{ZnO}$ showed greater potential to increase total root length, root area, root depth, root dry weight than did cultivar Julgeh which is more likely to invest more of its assimilate on shoot parameters instead of greater belowground investment. Similiarly, Subramanian et al. (2009) illustrated significant increases in morphological root traits including root length (vertical), root spread (horizontal), root volume and physiological parameters including chlorophyll content and grain $\mathrm{Zn}$ concentration in inoculated maize plants compared with un-inoculated plants and highlighted that this improvement was re-enforced with the application of $\mathrm{Zn}$ in the form of $\mathrm{ZnSO}_{4}$ fertilisation to the soil. Apart from the advantages of the mycorrhizal symbiosis, the increment in root traits with application of $\mathrm{Zn}$ may due to the vital role played by $\mathrm{Zn}$ in auxin synthesis. In recent observations of rice seedlings under $\mathrm{Zn}$ deficiency, auxin production decreased resulting in a significant reduction in morpho-physiological traits and also root growth (Begum et al., 2016). Our findings suggest that cultivar Yusuf with greater belowground investment particularly in vertical development of root traits such as total root length and root depth may be better able to take advantage for water and nutrient acquire from the deeper layers of the soil. Therefore, we hypothesis that cultivar Yusuf with its vertical root development 
and deeper root structure is better suited as a dryland cultivar in arid to semi-arid environments. Similarly, Koevoets et al. (2016) showed that crops with thinner and deeper root systems or higher root: shoot ratio are more likely to acquire more water from deeper soils, which could be an adaptation to alleviate the impact of drought during production of those crops.

We found that inoculation with $G$. mosseae highlighted a high capacity to increase horizontal development of the root system of cultivar Julgeh with improvements in root diameter and root volume. Feddermann et al. (2010) also observed that plants inoculated with AM fungi had reduced root: shoot biomass ratio due to allocation of more biomass to shoots. They concluded that this allometric relationship between roots and shoots increased carbon assimilation to meet the demand of the AM fungi (Feddermann et al., 2010). According to Nouri et al. (2014) there is a reciprocal relationship between the amount of elements such as $\mathrm{N}$ and $\mathrm{P}$ and fungal coexistence, so that deficiency in these two macro elements strengthens the symbiotic relationship and increases colonisation of the host plant. A low rate of root colonisation in nonmycorrhizal plants can be due to increased P (Liu et al., 2016). However, foliar application of $\mathrm{Zn}$ could address $\mathrm{Zn}$ deficiency caused by interactions between $\mathrm{Zn}$ and $\mathrm{P}$ in soil (Mousavi et al., 2011), and AM fungi could increase the efficiency of use of $P$ fertiliser. Thus, co-inoculation with AM fungi and ordinary foliar application of $\mathrm{ZnO}$ could be an agronomic practice for effective barley production and the nano form of $\mathrm{Zn}$ could contribute to higher $\mathrm{Zn}$ concentration in barley grain. The percentage of mycorrhizal colonisation has not been measured in this study.

\section{Conclusions}

The two cultivars of barley investigated here differed in response to combinations of the form of $\mathrm{Zn}$ applied as a foliar spray and inoculation with two commercial inocula of AM fungi. Foliar $\mathrm{Zn}$ application in the nano form significantly enhanced root and shoot morphological and physiological traits, and increased the concentration of $\mathrm{Zn}$ in barley grain. The barley cultivar Julgeh inoculated with $G$. mosseae had physiological traits that were more likely to enhance yield and yield parameters than those of barley cultivar Yusuf that invested more in root traits and vegetative growth. The extent of benefit of mycorrhizal inoculation for each barley cultivar depended on the form of foliar $\mathrm{Zn}$ applied. The unique approach of nano $\mathrm{Zn}$ in the form of foliar fertiliser could elevate low concentration of $\mathrm{Zn}$ in barley grain, ensure the quality and vigor of seedlings in $\mathrm{Zn}$-deficient soils, and eliminate the need for application of fertiliser $\mathrm{Zn}$ to the soil. Therefore, application of nano foliar fertiliser and exploitation of naturally occurring AM fungi have potential to contribute to sustainable agricultural production by reducing the requirement for application of synthetic chemical fertilisers. Hence, a plausible strategy would be to select appropriate levels and forms of $\mathrm{P}$ fertiliser based on soil and crop requirements. This would contribute to $\mathrm{P}$ management, nutrient use efficiency, and improved contributions from the microbial community in soil. Such a fertiliser management approach, along with the $\mathrm{Zn}$ spraying, could make a positive step towards increasing the content of $\mathrm{Zn}$ in grain and its quality. Overall, foliar $\mathrm{Zn}$ application and mycorrhizal inoculation could contribute to the quality and quantity of barley grains, especially under arid and semi-arid regions.

\section{References}

Andreini C, Banci L, Bertini I, Rosato A, 2006. Counting the zincproteins encoded in the human genome. J. Proteome Res. 5:196-201.

Barea JM, Pozo MJ, Azcón R, Azcón-Aguilar C, 2005. Microbial cooperation in the rhizosphere. J. Exp. Bot. 56:1761-78.

Barman H, Das SK, Roy A, 2018. Zinc in soil environment for plant health and management strategy. Univers. J. Agric. Res. 6:149-54

Barrientos L, Scott JJ, Murthy PP, 1994. Specificity of hydrolysis of phytic acid by alkaline phytase from lily pollen. Plant Physiol. 106:1489-95.

Begum MC, Islam M, Sarkar MR, Azad MAS, Huda AN, Kabir AH, 2016. Auxin signaling is closely associated with Zn-efficiency in rice (Oryza sativa L.). J. Plant Interact. 11:124-9.

Blanke V, Renker C, Wagner M, Fullner K, Held M, 2005. Nitrogen supply affects arbuscular mycorrhizal colonisation of Artemisia vulgaris in a phosphate-polluted field site. N Phytol. 166:981-92.

Brown PH, Cakmak I, Zhang Q, 1993. Zinc in soils and plants. In: Robson, ed. Developments in plant and soil sciences. Springer, Dordrecht, The Netherlands, pp. 93-106.

Cakmak I, 2008. Enrichment of cereal grains with zinc: Agronomic or genetic biofortification. Plant Soil. 302:1-17.

Cakmak I, 2009. Enrichment of fertilisers with zinc: An excellent investment for humanity and crop production in India. J. Trace Elem. Med. Biol. 23:281-9.

Chaab A, Savaghebi GR, Motesharezadeh B, 2011. Differences in the zinc efficiency among and within maize cultivars in a calcareous soil. Asian J. Agric. Sci. 3:26-31.

Chapman HD, Pratt PF, 1961. Methods of analysis for soils, plants and waters. Division of Agricultural Sciences, University of California, CA, USA.

Deshpande P, Dapkekar A, Oak M, Paknikar K, Rajwade J, 2018. Nanocarrier-mediated foliar zinc fertilisation influences expression of metal homeostasis related genes in flag leaves and enhances gluten content in durum wheat. PLoS One [epub ahead of print].

Dhawal S, Sarkar DR, Yadav RS, Parihar M, Rakshit A, 2016. Biopriming with arbuscular mycorrhizae for addressing soil fertility with special reference to phosphorus. Int. J. Bioresour. Sci. 3:35-40.

Eichert T, Kurtz A, Steiner U, Goldbach HE, 2008. Size exclusion limits and lateral heterogeneity of the stomatal foliar uptake pathway for aqueous solutes and water suspended nanoparticles. Physiol. Plantarum. 134:151-60.

Eleiwa E, Kord A, Ibrahim SA, 2013. Response of barley plants to foliar application of growth regulators mixture of indole acetic acid, naphthalene acetic acid and zinc. Afr. J. Biotechnol. 12:3653-61.

Feddermann N, Finlay R, Boller T, Elfstrand M, 2010. Functional diversity in arbuscular mycorrhiza the role of gene expression, phosphorus nutrition and symbiotic efficiency. Fungal Ecol. $3: 1-8$.

Fedorenko V, Buklagin D, Golubev I, Nemenushchaya L, 2015. Review of Russian nanoagents for crops treatment. Nanotechnol. Russia. 10:318-24.

Fileppi M, Galasso I, Tagliabue G, Daminati MG, Campion B, Doria E, Sparvoli F, 2010. Characterisation of structural genes involved in phytic acid biosynthesis in common bean (Phaseolus vulgaris L.). Mol Breeding. 25:453-70. 
Genc Y, McDonald GK, Graham RD, 2004. Differential expression of zinc efficiency during the growing season of barley. Plant Soil. 263:273-82.

Gibson T, Leece D, 1981. Estimation of physiologically active zinc in maize by biochemical assay. Plant Soil. 63:395-406.

Haslett BS, Reid RJ, Rengel Z, 2001. Zinc mobility in wheat: uptake and distribution of zinc applied to leaves or roots. Ann Bot. 87:379-86.

Imran M, Rehim A, Sarwar N, Hussain S, 2016. Zinc bioavailability in maize grains in response of phosphorous-zinc interaction. J. Plant Nutr. Soil Sci. 179:60-6.

Jalali M, 2007. Phosphorus status and sorption characteristics of some calcareous soils of Hamadan, western Iran. Environ Geol. 53:365-74.

Jan M, Anwar-ul-Haq M, Tanveer-ul-Haq AA, Wariach EA, 2016. Evaluation of soil and foliar applied $\mathrm{Zn}$ sources on rice (Oryza sativa L.) genotypes in saline environments. Int. J. Agricul. Biol. 18:643-8.

Janmohammadi M, Sabaghnia N, Dashti S, Nouraein M, 2016. Investigation of foliar application of nano-micronutrient fertilisers and nano-titanium dioxide on some traits of barley. Biologija. 62:148-56.

Joseph T, Morrisson M. 2006. Nanotechnology in agriculture and food. Available from: http://nanoforum.org

Joy EJ, Stein A, Young SD, Ander EL, Watts MJ, Broadley MR, 2015. Zinc-enriched fertilisers as a potential public health intervention in Africa. Plant Soil. 389:1-24.

Khan GA, Bouraine S, Wege S, Li Y, De Carbonnel M, Berthomieu P, Poirier Y, Rouached H, 2014. Coordination between zinc and phosphate homeostasis involves the transcription factor PHR1, the phosphate exporter PHO1, and its homologue PHO1; H3 in Arabidopsis. J. Exp. Bot. 65:871-84.

Kinaci G, Kinaci E. 2005. Effect of zinc application on quality traits of barley in semi arid zones of Turkey. Plant Soil. Environ. 51:328-34.

Koevoets IT, Venema JH, Elzenga JT, Testerink C, 2016. Roots withstanding their environment: exploiting root system architecture responses to abiotic stress to improve crop tolerance. Front Plant Sci. 7:1335.

Kothari S, Marschner H, Romheld V, 1991. Contribution of the VA mycorrhizal hyphae in acquisition of phosphorus and zinc by maize grown in a calcareous soil. Plant Soil. 131:177-85.

Kumar A, 2001. Studies on zinc use efficiency in wheat genotypes. PhD Dissertation, Division of Plant Physiology, New Delhi, India.

Liu W, Zhang Y, Jiang S, Deng Y, Christie P, Murray PJ, Zhang J, 2016. Arbuscular mycorrhizal fungi in soil and roots respond differently to phosphorus inputs in an intensively managed calcareous agricultural soil. Sci. Rep. 6:24902.

Mahajan P, Dhoke SK, Khanna AS, 2011. Effect of nano-ZnO particle suspension on growth of mung (Vigna radiata) and gram (Cicer arietinum. L) seedlings using plant agar method. J. Nanotechnol. 2011:7.

Morales-Diaz AB, Ortega-Ortiz H, Juarez-Maldonado A, CadenasPliego G, Gonzalez-Morales S, Benavides-Mendoza A, 2017. Application of nanoelements in plant nutrition and its impact in ecosystems. Adv. Nat. Sci. Nanosci. Nanotechnol. 8:013001.

Mousavi SR, 2011. Zinc in crop production and interaction with phosphorus. Aust. J. Basic Appl. Sci. 5:1503-9.

Naderi MR, Danesh-Shahraki A, 2013. Nanofertilisers and their roles in sustainable agriculture. Int. J. Agri. Crop Sci. 5:2229-32.

Nouri E, Breuillin-Sessoms F, Feller U, Reinhardt D, 2014. Phosphorus and nitrogen regulate arbuscular mycorrhizal sym- biosis in Petunia hybrida. PLoS One. 9:e90841.

Ova EA, Kutman UB, Ozturk L, Cakmak I, 2015. High phosphorus supplyreduced zinc concentration of wheat in native soil but not in autoclaved soil ornutrient solution. Plant Soil. 393:147-62.

Paech K, Simonis W, 1952. Pflanzenphysiologische Praktika, Springer Verlag, Berlin Göttingen Heidelberg, Germany.

Painkra B, Srivastava LK, Chandel G, 2015. Effect of Zn application on different rice genotypes in yield, $\mathrm{Zn}$ content and $\mathrm{Zn}$ uptake. Ecoscan. 7:251-8.

Pellegrino E, Öpik M, Bonari E, Ercoli L, 2015. Responses of wheat to arbuscular mycorrhizal fungi: a metaanalysis of field studies from 1975 to 2013. Soil Biol. Biochem. 84:210-7.

Porra R, Thompson W, Kriedemann P, 1989. Determination of accurate extinction coefficients and simultaneous equations for assaying chlorophylls a and b extracted with four different solvents: verification of the concentration of chlorophyll standards by atomic absorption spectroscopy. Biochim. Biophys. Acta Bioenerg. 975:384-94.

Prasad TNVKV, Sudhakar P, Sreenivasulu Y, Latha P, Munaswamy V, Raja Reddy K, Sreeprasad TS, Sajanlal PR, Pradeep T, 2012. Effect of nanoscale zinc oxide particles on the germination, growth and yield of peanut. J. Plant. Nutr. 35:905-27.

Rehman H, Aziz T, Farooq M, Wakeel A, Rengel Z, 2012. Zinc nutrition in rice production systems: a review. Plant Soil. 361:203-26.

Rengel Z, 1995. Carbonic anhydrase activity in leaves of wheat genotypes differing in Zn efficiency. J. Plant Physiol. 147:251-6.

Rengel Z, Romheld V, 2000. Root exudation and Fe uptake and transport in wheat genotypes differing in tolerance to $\mathrm{Zn}$ deficiency. Plant Soil. 222:25-34.

Sadeghzadeh B, 2013. A review of zinc nutrition and plant breeding. J. Soil Sci. Plant. Nutr. 13:905-27.

Singh B, Natesan SKA, Singh BK, Usha K, 2005. Improving zinc efficiency of cereals under zinc deficiency. Curr Sci. 10:36-44.

Singh B, Singh BK, 2011. Effect of reduced seed and applied zinc on zinc efficiency of wheat genotypes under zinc deficiency in nutrient solution culture. J. Plant Nutr. 34:449-64.

Singh MD, Gautam C, Patidar OP, Meena HM, 2017. Nano fertilisers is a new way to increase nutrients use efficiency in crop production. Int. J. Agr. Sci. 9:3831-3.

Smith FA, Grace EJ, Smith SE, 2009. More than a carbon economy: nutrient trade and ecological sustainability in facultative arbuscular mycorrhizal symbioses. N. Phytol. 182:347-58.

Subbaiah LV, Prasad TNVKV, Krishna TG, Sudhakar P, Reddy BR, Pradeep T, 2016. Novel effects of nanoparticulate delivery of zinc on growth, productivity, and zinc biofortification in maize (Zea mays L.). J. Agric. Food Chem. 64:3778-88.

Subramanian KS, Tenshia JS, Jayalakshmi K, Ramachandran V, 2009. Role of arbuscular mycorrhizal fungus (Glomus intraradices) in zinc nutrition of maize. J. Agric. Biotech. Sustain. Develop. 1:29-38.

Sundaram S, Stalin P, 2016. Screening of zinc efficient and inefficient rice genotypes in low soil status. Bioscan. 11:2637-43.

Tarafdar JC, Raliya R, Mahawar H, Rathore I, 2014. Development of zinc nanofertiliser to enhance crop production in pearl millet (Pennisetum americanum L). Agric. Res. 3:257-62.

Teng W, Deng Y, Chen XP, Xu XF, Chen RY, Lv Y, Zhao YY, Zhao $\mathrm{XQ}, \mathrm{He} \mathrm{X}, \mathrm{Li} \mathrm{B}, 2013$. Characterisation of root responseto phosphorus supply from morphology to gene analysis in fieldgrown wheat. J. Exp. Bot. 64:1403-11.

Thompson JP, Clewett TG, Fiske ML, 2013. Field inoculation witharbuscular-mycorrhizal fungi overcomes phosphorus and 
zinc deficiencies of linseed (Linum usitatissimum.L) in a vertisol subject to long-fallow disorder. Plant Soil. 371:117-37.

Tiong J, McDonald G, Genc Y, Shirley N, Langridge P, Huang CY, 2015. Increased expression of six ZIP family genes by zinc (Zn) deficiency is associated with enhanced uptake and rootto-shoot translocation of $\mathrm{Zn}$ in barley (Hordeum vulgare). N. Phytol. 207:1097-109.

Velu G, Ortiz-Monasterio I, Cakmak I, Hao Y, Singh R, 2014. Biofortification strategies to increase grain zinc and iron concentrations in wheat. J. Cereal. Sci. 59:365-72.

Wang WN, Tarafdar JC, Biswas P, 2013. Nanoparticle synthesis and delivery by an aerosol route for watermelon plant foliar uptake. J. Nanopart. Res. 15:1417.

Yagmur M, Arpali D, Gulser F, 2017. Effects of zinc and urea as foliar application on nutritional properties and grain yield in barley (Hordeum vulgare 1. Conv. Distichon) under semi-arid condition. Fresen. Environ. Bull. 26:6085-92.
Yang J, Zhang J, Wang Z, Zhu O, Wang W, 2001. Remobilisation of carbon reserves in response to water deficit during grain filling of rice. Field Crop Res. 71:47-55.

Zhang W, Liu D, Liu Y, Cui Z, Chen X, Zou C, 2016. Zinc uptake and accumulation in winter wheat relative to changes in root morphology and mycorrhizal colonisation following varying phosphorus application on calcareous soil. Field Crop Res. 197:74-82.

Zhang YQ, Deng Y, Chen RY, Cui ZL, Chen XP, Yost R, Zhang FS, Zou CQ, 2012a. The reduction in zinc concentration of wheat grain upon increased phosphorus-fertilisation and its mitigation by foliar zinc application. Plant Soil. 361:143-52.

Zhang YQ, Sun YX, Ye YL, Karim MR, Xue YF, Yan P, Meng OF, Cui ZL, Cakmak I, Zhang FS, 2012b. Zinc biofortification of wheat through fertiliser applications in different locations of China. Field Crop Res. 125:1-7. 